\title{
Thermomechanical deformations in photovoltaic laminates
}

\author{
M Paggi $^{1 *}$, S Kajari-Schröder ${ }^{2}$, and U Eitner ${ }^{2}$ \\ ${ }^{1}$ Institut für Kontinuumsmechanik, Leibniz Universität Hannover, Hannover, Germany. On leave from Politecnico di \\ Torino, Italy \\ ${ }^{2}$ Institute for Solar Energy Research Hamelin, Emmerthal, Germany
}

The manuscript was received on 5 April 2011 and was accepted after revision for publication on 8 August 2011.

DOI: $10.1177 / 0309324711421722$

\begin{abstract}
Recent experimental results based on the digital image correlation technique (U. Eitner, M. Köntges, R. Brendel, Solar Energy Mater. Solar Cells, 2010, 94, 1346-1351) show that the gap between solar cells embedded into a standard photovoltaic laminate varies with temperature. The variation of this gap is an important quantity to assess the integrity of the electric connection between solar cells when exposed to service conditions. In this paper, the thermo-elastic deformations in photovoltaic laminates are analytically investigated by developing different approximate models based on the multilayered beam theory. It is found that the temperature-dependent thermo-elastic properties of the encapsulating polymer layer are responsible for the deviation from linearity experimentally observed in the diagram relating the gap variation to the temperature. The contribution of the different material constituents to the homogenized elastic modulus and thermal expansion coefficient of the composite system is also properly quantified through the definition of weight factors of practical engineering use.
\end{abstract}

Keywords: photovoltaic laminate, multilayered system, thermo-elastic deformations, analytical model, digital image correlation

\section{INTRODUCTION}

Photovoltaics (PV) is the direct conversion of solar radiation into electricity using semiconductors that exhibit the photovoltaic effect. Photovoltaic power generation employs solar panels comprising a number of silicon solar cells embedded in laminates. Owing to the growing demand for renewable energy sources, the cumulative installed photovoltaic power has increased from $1.4 \mathrm{GW}$ in 2000 to $22.9 \mathrm{GW}$ in 2009 [1] while the cost per $\mathrm{kW}_{\text {peak }}$ has declined.

Typical crystalline silicon PV laminates are multilayered structures composed of a glass superstrate, the interconnected silicon (Si) solar cells, an

*Corresponding author: Faculty of Engineering, Politecnico di Torino, Corso Duca degli Abruzzi, 24, Torino 10129, Italy. email:paggi@ikm.uni-hannover.de,marco.paggi@polito.it encapsulating polymer (ethylene vinyl acetate, EVA), and a polymeric protective backsheet. Figure 1 shows the schematic composition of such a PV module. A typical distance between neighbouring solar cells is $2 \mathrm{~mm}$, a small quantity as compared to the span of a solar cell of either 125 or $156 \mathrm{~mm}$. The gap between solar cells was recently found to be dependent on temperature [2]. It varies up to $60 \mu \mathrm{m}$ within the temperature range of $-40^{\circ} \mathrm{C}$ and $85^{\circ} \mathrm{C}$, which is representative for the temperature range that $\mathrm{PV}$ modules experience in the field $[3,4]$. The electrical connection between the cells needs to withstand these deformations through many thermal cycles without failure. Therefore it is imperative to understand and quantify the relative displacement of the solar cells within the laminate.

The challenge in understanding the gap deformation in the PV modules stems from the fact that both the mechanical properties and the dimensions 
of the different materials in the PV module differ considerably from each other. The glass superstrate, with a thickness of about $4 \mathrm{~mm}$, is an order of magnitude thicker than the other layers, and thus dominates the deformation of the PV module. The thermal expansion coefficients vary between the different materials up to two orders of magnitude, leading to considerable thermal stresses. Finally, the stiffness of the constituents range over up to four orders of magnitude. Temperature-dependent mechanical properties of EVA add further complexity to the problem.

The computation of thermo-elastic deformations in PV laminates is a prerequisite to propose new technical solutions that may result in innovative solar cell design. In this context, the development of analytical models would be convenient to understand the roles played by the different materials on the global mechanical behaviour.

The analysis of thermo-elastic stresses in composite beams with homogeneous dissimilar layers can be traced back to the pioneering work of Timoshenko [5]. On the basis of an elementary beam theory he determined the normal stresses in bilayered beams, assuming that these stresses remain unchanged along the longitudinal beam axis, i.e. considering a perfectly bonded bi-material interface. Afterwards, various simplified approaches to the problem in question were put forward, most in connection with the needs of micro-electronics technology. Suhir [6, 7] extended the Timoshenko solution by considering deformable interfaces. Introducing both the longitudinal and the transverse interfacial compliances, he evaluated the magnitude and distribution of the shearing and peeling stresses along the interface of bi-metal thermostats. More recently, the Suhir solution was improved in reference [8], where a correction to the peeling stresses was proposed in order to satisfy the translation equilibrium in the direction normal to the layers. The discrepancies between the Suhir solution and the finite-element results were also analysed in reference [9], where further corrections were proposed. An extension of this approach to electronic assemblies composed of three layers was proposed in reference [10], although the thickness of the intermediate layer, i.e. the adhesive one, was considered much smaller than those of the adjacent layers. A general theory for the analysis of interfacial stresses in multi-layered homogeneous beams was recently discussed in references [11] and [12], although the applications regarded electronic packaging with three layers only. Finally, Carpinteri and Paggi [13] proposed a general theory for functionally graded multilayered beams with shear deformable interfaces and for a general number of layers.

The application of such theories to PV laminates is however not straightforward at all. All the available models assume constant material properties for the layers along the longitudinal (axial) direction. This is not the case of PV laminates, where the solar cells are longitudinally separated by EVA. Moreover, all the models listed above assume temperature independent material properties, which is not realistic for polymer layers that exhibit a strong temperature-dependent behaviour.

Hence, in the present work, the theory of multilayered beams will be extended to the thermoelastic analysis of PV laminates. The non-continuity of the solar cells, separated by EVA, will be carefully modelled. Different assumptions will be tested in order to propose a closed-form solution for the computation of the gap between solar cells as a function of temperature, keeping the formulation as simple as possible. The resulting models' predictions will be compared to the experimental measurements carried out in the Institute for Solar Energy Research in Hamelin (ISFH). As a main result, the role played by the individual material constituents on the overall deformability of the PV laminate is elucidated.

\section{MATERIAL PROPERTIES AND GEOMETRY}

Consider a multilayered material sketched in Fig. 1, where it is possible to identify three structural components making a representative element of a PV panel, periodically repeated along the longitudinal (axial) coordinate: a glass superstrate with thickness $h_{\mathrm{G}}=4 \mathrm{~mm}$, and two composite beams. Beam 1 has 4 layers: an encapsulating polymer layer (EVA) with thickness $h_{\mathrm{EVA}}=0.5 \mathrm{~mm}$, the silicon solar cell with thickness $h_{\mathrm{Si}}=0.166 \mathrm{~mm}$, another layer of EVA

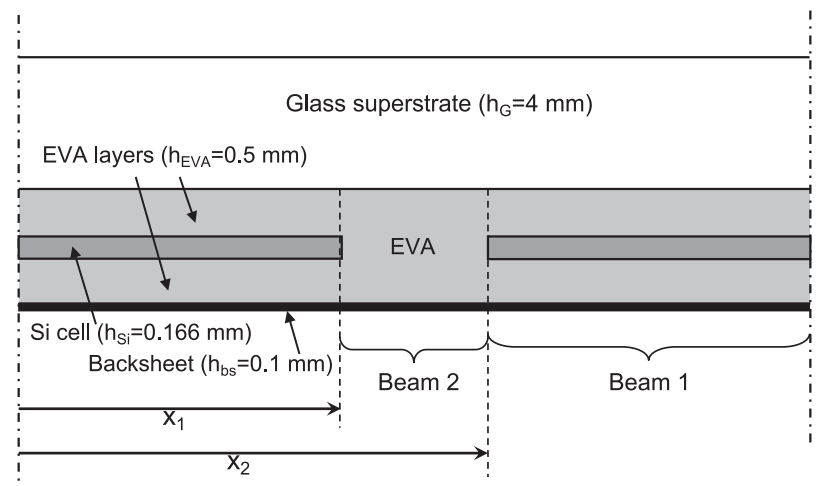

Fig. 1 Sketch of a representative element of a PV module (not in scale); the variables are defined in the Appendix 
Table 1 Values of the geometrical and mechanical parameters

\begin{tabular}{ll}
\hline Variable & Value \\
\hline Geometrical parameters & \\
$h_{\mathrm{bs}}$ & $0.1 \mathrm{~mm}$ \\
$h_{\mathrm{EVA}}$ & $0.5 \mathrm{~mm}$ \\
$h_{\mathrm{G}}$ & $4 \mathrm{~mm}$ \\
$h_{\mathrm{Si}}$ & $0.166 \mathrm{~mm}$ \\
$l_{1,0}$ & $125 \mathrm{~mm}$ \\
$l_{2,0}$ & $2 \mathrm{~mm}$ \\
$\mathrm{Mechanical} \mathrm{parameters}$ & \\
$E_{\mathrm{bs}}$ & $2.8 \mathrm{GPa}$ \\
$E_{\mathrm{EVA}}$ & dependent on $T$, see Fig. 2 \\
$E_{\mathrm{G}}$ & $73 \mathrm{GPa}$ \\
$E_{\mathrm{Si}}$ & $130 \mathrm{GPa}$ \\
$\alpha_{\mathrm{bs}}$ & $5.04 \times 10^{-5}$ \\
$\alpha_{\mathrm{EVA}}$ & $2.70 \times 10^{-4}$ \\
$\alpha_{\mathrm{G}}$ & $8.00 \times 10^{-6}$ \\
$\alpha_{\mathrm{Si}}$ & $2.49 \times 10^{-6}$ \\
\hline
\end{tabular}

with the same thickness as the previous one, and finally a thin backsheet made of an ethylene tetrafluoroethylene core with silicon nitride coating (isovolta Icosolar T 2754), with thickness $h_{\mathrm{bs}}=0.1 \mathrm{~mm}$. The span of beam 1 at $20^{\circ} \mathrm{C}, l_{1,0}$, is $125 \mathrm{~mm}$. Beam 2 is composed of two layers: an EVA layer with total thickness equal to $h_{\mathrm{Si}}+2 h_{\mathrm{EVA}}$, and the backsheet as in beam 1 . Its span $l_{2,0}$ is equal to $2 \mathrm{~mm}$ at $20^{\circ} \mathrm{C}$. All the values of the geometrical parameters are summarized in Table 1.

Not only are the thicknesses of the layers quite different from each other, but also the mechanical properties of the constituent materials differ considerably. As a result, although some layers are quite thin, it is not obvious if their mechanical contribution to the deformation field can be neglected. More specifically, considering the Young's moduli, glass and silicon are much stiffer than backsheet and EVA. In fact $E_{\mathrm{G}}=73 \mathrm{GPa}[14], E_{\mathrm{Si}}=130 \mathrm{GPa}$ [15], and $E_{\mathrm{bs}}=2.8 \mathrm{GPa}$. The value for the backsheet is determined from tensile tests using ASTM D638 TYPE I specimens and a strain velocity of $0.175 / \mathrm{min}$. The Young's modulus is calculated by linear regression of the true-stress to true-strain curve in the strain interval 0.001-0.015.

The Young's modulus of EVA is strongly dependent on temperature and varies from $1 \mathrm{MPa}$ at high temperatures to $1 \mathrm{GPa}$ at low temperatures. To better characterize this material, tensile relaxation tests have been performed in ISFH [16] in order to determine $E_{\mathrm{EVA}}$ versus temperature for different relaxation times. Here, it is possible to use the isothermal relaxation curves given in reference [16] to create isochronal curves over temperature. Times of $1 \mathrm{~s}, 2 \mathrm{~s}, 5 \mathrm{~s}, 10 \mathrm{~s}$, $20 \mathrm{~s}, 1 \mathrm{~min}, 2 \mathrm{~min}, 5 \mathrm{~min}, 10 \mathrm{~min}, 20 \mathrm{~min}, 1 \mathrm{~h}$, and $2 \mathrm{~h}$ in the isothermal experimental data were selected and the corresponding value of the tensile relaxation

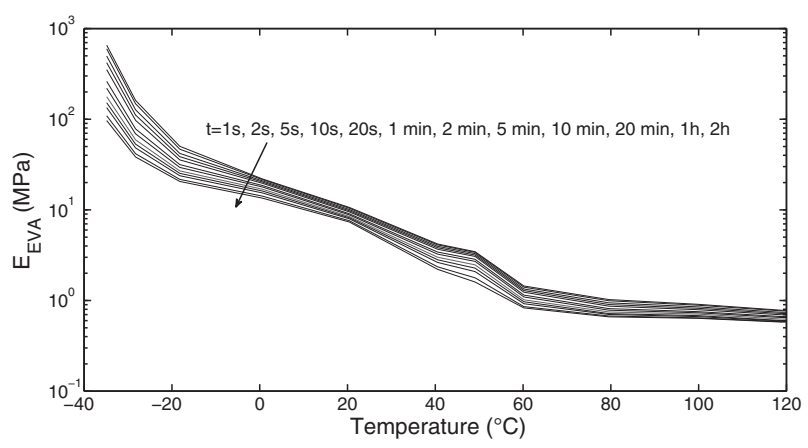

Fig. 2 Elastic modulus of EVA versus temperature, for different relaxation times

modulus read out. These moduli are then plotted versus temperature as shown in Fig. 2. For high temperatures, the Young's modulus of EVA tends to approximately $1 \mathrm{MPa}$ regardless of the relaxation time. This value is nearly five orders of magnitude lower than that of glass and silicon, and three orders of magnitude lower than that of backsheet. On the other hand, for low temperatures, the elastic modulus of EVA increases considerably and ranges from 0.1 to $1 \mathrm{GPa}$, depending on the relaxation time.

Regarding the thermal expansion coefficients, the situation is reverted and glass and silicon present the lowest values. Typical values for float glass are in the range of $7.5 \times 10^{-6}$ to $9 \times 10^{-6} / \mathrm{K}$. An intermediate value of $\alpha_{\mathrm{G}}=8.00 \times 10^{-6}$ is used in the calculation here. For silicon, $\alpha_{\mathrm{Si}}=2.5 \times 10^{-6}$ was selected, as determined in reference [17]. EVA has a thermal expansion coefficient approximately two orders of magnitude higher, $\alpha_{\mathrm{EVA}}=2.70 \times 10^{-4}$, which was determined from the tensile elongation during a dynamical mechanical analysis in torsion. The manufacturer of the backsheet gives $\alpha_{\mathrm{bs}}=5.04 \times 10^{-5}$, which is an intermediate value between glass and silicon on one hand and EVA on the other hand. All the values of the material parameters are summarized in Table 1.

Owing to such a significant mismatch in the thermo-elastic properties of the constituent materials, a complex state of stress and deformation is expected in the PV laminate. In order to gain some insight into the interplay of the materials and their impact on the gap deformation, an analytical model is developed in the next section for the prediction of the gap deformation in PV laminates.

\section{ANALYTICAL MODEL FOR THE COMPUTATION OF THE GAP BETWEEN SOLAR CELLS}

Specific simplifications of the complicated mechanical interplay of the different mechanical properties 
in a PV laminate allow the development of an analytical model. Therewith the computation of the gap between solar cells in PV laminates can be performed as a function of the environmental temperature.

\subsection{Derivation of the equivalent mechanical parameters}

The central simplification within this work is to assume shear-free deformation in the complete system. This leads to a quasi-one-dimensional (1D) analysis, thus drastically reducing the degrees of freedom and allowing for a comprehensible analytical solution. It is, however, not a particularly good assumption for the EVA at least at high temperatures, as it is much more compliant than the other materials and will therefore shear to compensate for the different thermal expansions of glass, silicon and backsheet. Instead of explicitly considering the shear deformation of EVA, it is assumed that the interface between glass and EVA is totally debonded, while all other interfaces are assumed to be perfectly bonded.

The influence of the underlying stack on the expansion of the glass is negligible, as the glass is much thicker than the other materials combined, and is second in stiffness only to the thin silicon. On the other hand, the influence of the glass on the remaining stack is considered in form of boundary conditions: the length of the remaining stack at any given temperature is determined by the freely expanded glass. This pinning of the centres of the solar cells to certain positions of the glass corresponds to symmetry conditions within the extended PV module.

These kinematic hypotheses allow the present authors to treat the problem in one dimension. Owing to its periodicity in the in-plane directions, the analysis is restricted to a representative PV laminate element, composed of two half-cells and an interlayer, as sketched in Fig. 1.

Under such conditions, attention is focused on the beams 1 and 2 composing the stack. Let an axial strain $\epsilon$ be imposed to the $i$ th beam $(i=1,2)$, uniform along its cross-section. The resulting axial forces in the beams are

$$
N_{i}=\sum_{j=1}^{n} \sigma_{j} h_{j} t=\epsilon \sum_{j=1}^{n} E_{j} h_{j} t
$$

where $t$ is the beam width, whereas $E_{j}$ and $h_{j}$ are the elastic moduli and the height of the $j$ th layer, respectively. The summation is extended to all the $n$ layers composing the $i$ th beam. The existence of an equivalent homogenous beam with the same mechanical response can now be postulated. Its behaviour in terms of axial force versus strain would be

$$
N_{i}=\epsilon E_{i}^{*} h t
$$

where $h=\sum_{j=1}^{n} h_{j}$ and $E_{i}^{*}$ are the composite elastic moduli. Equating equations (1) and (2), the expression for $E_{i}^{*}$ is obtained, which coincides with the Voigt estimate for Young's modulus of a composite system under isostrain conditions and perfectly bonded bi-material interfaces $[\mathbf{1 8}]$

$$
E_{i}^{*}=\frac{\sum_{j=1}^{n} E_{j} h_{j}}{\sum_{j=1}^{n} h_{j}}
$$

Equation (3) implicitly assumes that the stress-strain constitutive relation of the material components is linear elastic. This assumption might not hold for EVA in the high-temperature regime. However, the elastic modulus of EVA is particularly low at those temperatures as compared to the elastic modulus of the other materials. Therefore, the error in using a linearized stress-strain curve of EVA instead of a strain-dependent Young's modulus is negligible. More specifically, for the problem here find

$$
\begin{aligned}
& E_{1}^{*}=\frac{2 E_{\mathrm{EVA}} h_{\mathrm{EVA}}+E_{\mathrm{Si}} h_{\mathrm{Si}}+E_{\mathrm{bs}} h_{\mathrm{bs}}}{h} \\
& E_{2}^{*}=\frac{E_{\mathrm{EVA}}\left(2 h_{\mathrm{EVA}}+h_{\mathrm{Si}}\right)+E_{\mathrm{bs}} h_{\mathrm{bs}}}{h}
\end{aligned}
$$

It is now possible to define the following stiffness weight factors to understand the contribution of each material constituent on the elasticity of the composite beam 1

$$
\begin{aligned}
f_{1}^{\mathrm{EVA}} & =\frac{2 E_{\mathrm{EVA}} h_{\mathrm{EVA}}}{h E_{1}^{*}} \\
f_{1}^{\mathrm{Si}} & =\frac{E_{\mathrm{Si}} h_{\mathrm{Si}}}{h E_{1}^{*}} \\
f_{1}^{\mathrm{bs}} & =\frac{E_{\mathrm{bs}} h_{\mathrm{bs}}}{h E_{1}^{*}}
\end{aligned}
$$

and the same for beam 2

$$
\begin{aligned}
f_{2}^{\mathrm{EVA}} & =\frac{E_{\mathrm{EVA}}\left(2 h_{\mathrm{EVA}}+h_{\mathrm{Si}}\right)}{h E_{2}^{*}} \\
f_{2}^{\mathrm{bs}} & =\frac{E_{\mathrm{bs}} h_{\mathrm{bs}}}{h E_{2}^{*}}
\end{aligned}
$$

These weight factors are plotted in Fig. 3, selecting an elastic modulus for EVA corresponding to a 


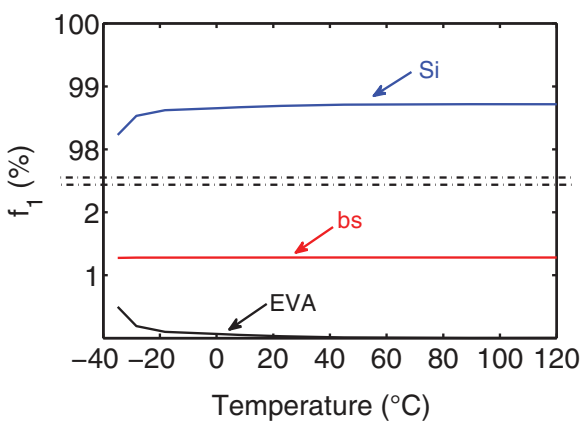

(a) Composite beam 1

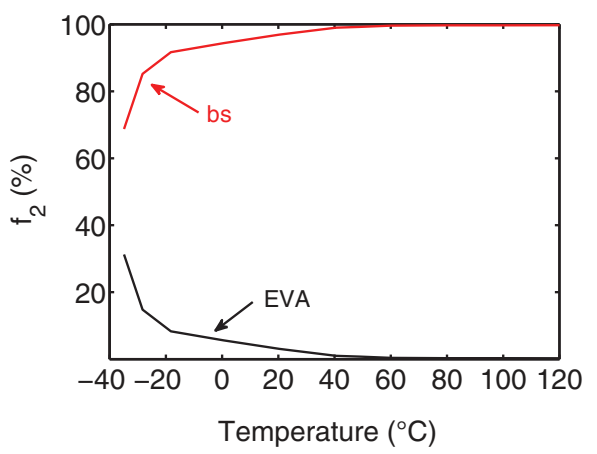

(b) Composite beam 2

Fig. 3 Weight factors for the composite Young's modulus; the labels (bs) and (Si) denote, respectively, the backsheet and the silicon cell

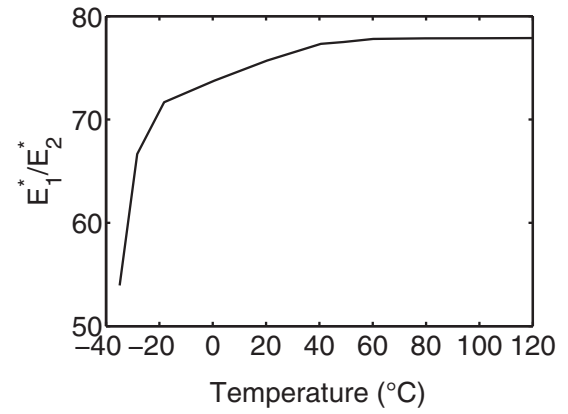

Fig. 4 Ratio between the composite elastic moduli of beams 1 and 2

relaxation time of $1 \mathrm{~h}$. This choice is consistent with the experimental conditions used to measure the variation of the gap between solar cells where the temperature is slowly raised and lowered as described in reference [2].

Figure 3 clearly shows that, under the condition of perfectly bonded interfaces, the axial stiffness of the composite beam 1 is substantially governed by the elastic modulus of the silicon cell. On the other hand, the stiffness of beam 2 is mostly governed by the backsheet for temperatures higher than $20^{\circ} \mathrm{C}$. For temperatures below $0^{\circ} \mathrm{C}$, the contribution of EVA significantly increases up to 30 per cent, due to its temperature-dependent elastic properties.

The ratio between the composite elastic moduli of beams 1 and 2 is shown in Fig. 4. This ratio is a non-linear function of temperature, again due to the temperature-dependent elastic modulus of EVA.

In a similar way, the composite thermal expansion coefficients can be determined. Neglecting the beam curvature due to the constraint exerted by the glass superstrate, the axial thermo-elastic stress in the $j$ th layer is [13]

$$
\sigma_{j}=-\alpha_{j} E_{j} \Delta T+\epsilon_{i} E_{j}
$$

where $\alpha_{j}$ is the thermal expansion coefficient of that layer. The axial deformation $\epsilon$ of the composite beam can now be determined by imposing the condition of vanishing axial force in a generic cross-section

$$
N=\sum_{j=1}^{n} \int_{y_{j}^{(2)}}^{y_{j}^{(1)}} \sigma_{j} t \mathrm{~d} y=0
$$

where $y_{j}^{(1)}$ and $y_{j}^{(2)}$ correspond, respectively, to the coordinates of the upper and lower interfaces of the $j$ th layer, their difference being equal to the layer thickness, $h_{j}$. The variable $t$ is the beam width, as defined in equation (1). Introducing equation (5) into (4) and solving for $\epsilon$ you get

$$
\epsilon_{i}=\frac{\sum_{j=1}^{n} \alpha_{j} E_{j} h_{j}}{\sum_{j=1}^{n} E_{j} h_{j}} \Delta T
$$

The existence of an equivalent homogenized beam with the same mechanical response can be postulated. Its thermo-elastic behaviour would be governed by

$$
\epsilon_{i}=\alpha_{i}^{*} \Delta T
$$

Equating equations (7) and (6) the expression for $\alpha_{i}^{*}$ is obtained as

$$
\alpha_{i}^{*}=\frac{\sum_{j=1}^{n} \alpha_{j} E_{j} h_{j}}{\sum_{j=1}^{n} E_{j} h_{j}}
$$

The following thermal expansion weight factors can be defined to understand the contribution of each 
material constituent to the composite thermal expansion coefficient of beam 1

$$
\begin{aligned}
g_{1}^{\mathrm{EVA}} & =\frac{2 E_{\mathrm{EVA}} \alpha_{\mathrm{EVA}} h_{\mathrm{EVA}}}{2 E_{\mathrm{EVA}} \alpha_{\mathrm{EVA}} h_{\mathrm{EVA}}+E_{\mathrm{Si}} \alpha_{\mathrm{Si}} h_{\mathrm{Si}}+E_{\mathrm{bs}} \alpha_{\mathrm{bs}} h_{\mathrm{bs}}} \\
g_{1}^{\mathrm{Si}} & =\frac{E_{\mathrm{Si}} \alpha_{\mathrm{Si}} h_{\mathrm{Si}}}{2 E_{\mathrm{EVA}} \alpha_{\mathrm{EVA}} h_{\mathrm{EVA}}+E_{\mathrm{Si}} \alpha_{\mathrm{Si}} h_{\mathrm{Si}}+E_{\mathrm{bs}} \alpha_{\mathrm{bs}} h_{\mathrm{bs}}} \\
g_{1}^{\mathrm{bs}} & =\frac{E_{\mathrm{bs}} \alpha_{\mathrm{bs}} h_{\mathrm{bs}}}{2 E_{\mathrm{EVA}} \alpha_{\mathrm{EVA}} h_{\mathrm{EVA}}+E_{\mathrm{Si}} \alpha_{\mathrm{Si}} h_{\mathrm{Si}}+E_{\mathrm{bs}} \alpha_{\mathrm{bs}} h_{\mathrm{bs}}}
\end{aligned}
$$

and of beam 2

$$
\begin{aligned}
g_{2}^{\mathrm{EVA}} & =\frac{E_{\mathrm{EVA}} \alpha_{\mathrm{EVA}}\left(2 h_{\mathrm{EVA}}+h_{\mathrm{Si}}\right)}{E_{\mathrm{EVA}} \alpha_{\mathrm{EVA}}\left(2 h_{\mathrm{EVA}}+h_{\mathrm{Si}}\right)+E_{\mathrm{bs}} \alpha_{\mathrm{bs}} h_{\mathrm{bs}}} \\
g_{2}^{\mathrm{bs}} & =\frac{E_{\mathrm{bs}} \alpha_{\mathrm{bs}} h_{\mathrm{bs}}}{E_{\mathrm{EVA}} \alpha_{\mathrm{EVA}}\left(2 h_{\mathrm{EVA}}+h_{\mathrm{Si}}\right)+E_{\mathrm{bs}} \alpha_{\mathrm{bs}} h_{\mathrm{bs}}}
\end{aligned}
$$

These weight factors are plotted in Fig. 5 for $E_{\mathrm{EVA}}$ corresponding to a relaxation time of $1 \mathrm{~h}$. The composite thermal expansion coefficient of beam 1 is mostly governed by silicon, which has a weight factor ranging from 60 per cent to 80 per cent, depending on the temperature. The backsheet contributes to 20 per cent, more or less independently from the temperature. The EVA has a non-linear contribution which is approximately negligible for temperatures higher than $60^{\circ} \mathrm{C}$ and become relevant for temperatures below $0^{\circ} \mathrm{C}$. Regarding beam 2, the contribution of EVA is negligible for temperatures higher than $60^{\circ} \mathrm{C}$, whereas it significantly increases for $T<0^{\circ} \mathrm{C}$, up to nearly 80 per cent.

The ratio between the composite thermal expansion coefficients of beams 1 and 2 is reported in Fig. 6. This ratio ranges between 0.04 and 0.06 in the considered temperature range.

\subsection{Computation of the variation of the gap between solar cells}

When a uniform temperature excursion $\Delta T$ is applied from the reference temperature, the spans $l_{1}, l_{2}$, and $l_{\mathrm{G}}$ modify as follows

$$
\begin{aligned}
& l_{1}=l_{1,0}\left(1+\alpha_{1}^{*} \Delta T\right) \\
& l_{2}=l_{2,0}\left(1+\alpha_{2}^{*} \Delta T\right) \\
& l_{\mathrm{G}}=l_{\mathrm{G}, 0}\left(1+\alpha_{\mathrm{G}} \Delta T\right)
\end{aligned}
$$

where $l_{1,0}, l_{2,0}$, and $l_{G, 0}$ are the initial spans corresponding to $\Delta T=0$, and are equal to $62.5 \mathrm{~mm}$, $2 \mathrm{~mm}$, and $127 \mathrm{~mm}$, respectively. According to the previous kinematic assumption, the total deformed span of the composite beam 1-2 must be equal to the span of the glass superstrate, for each temperature value. In formulae $x_{1}(\Delta T)+x_{2}(\Delta T)=l_{\mathrm{G}}(\Delta T)$ where $x_{1}$ and $x_{2}$ are the equilibrium coordinates of the interfaces between beams 1 and 2 (see Fig. 1). This boundary condition allows the unknown $x_{2}(\Delta T)$ to be written in terms of $x_{1}(\Delta T)$

$$
x_{2}=l_{\mathrm{G}}-x_{1}
$$

Under the assumption of no external loads and purely axial deformation of the stack, the total potential energy of the system coincides with the deformation energy. For Clapeyron's theorem

$$
\mathcal{E}_{\mathrm{tot}}=2 \frac{E_{1}^{*}}{2}\left(\frac{x_{1}-l_{1}}{l_{1}}\right)^{2}+\frac{E_{2}^{*}}{2}\left(\frac{x_{2}-x_{1}-l_{2}}{l_{2}}\right)^{2}
$$

where the terms in parentheses correspond to the axial strains of the composite beams 1 and 2, respectively. Again, in the derivation, linear elastic stress-strain constitutive relations for the materials have been assumed. The error in using a linearized stress-strain curve of EVA at high temperatures instead of a strain-dependent Young's modulus is reasonably negligible.

The equilibrium position $x_{1}$ is now determined of the interface between beams 1 and 2 introducing equation (10) into (11), and finding the minimum of the total energy

$$
\frac{d \mathcal{E}_{\mathrm{tot}}\left(x_{1}\right)}{d x_{1}}=2 E_{1}^{*} \frac{x_{1}-l_{1}}{l_{1}^{2}}-2 E_{2}^{*} \frac{l_{\mathrm{G}}-2 x_{1}-l_{2}}{l_{2}^{2}}=0
$$

This gives

$$
x_{1}=\frac{E_{1}^{*} l_{1} l_{2}^{2}+E_{2}^{*} l_{1}^{2}\left(l_{\mathrm{G}}-l_{2}\right)}{l_{2}^{2} E_{1}^{*}+2 l_{1}^{2} E_{2}^{*}}
$$

and the variation of the gap between the solar cells is finally given by

$$
\begin{aligned}
& \Delta g=\left(x_{2}-x_{1}\right)-l_{2,0}=l_{\mathrm{G}}-2 x_{1}-l_{2,0}=l_{\mathrm{G}}-l_{2,0} \\
& -2 \frac{E_{1}^{*} l_{1} l_{2}^{2}+E_{2}^{*} l_{1}^{2}\left(l_{\mathrm{G}}-l_{2}\right)}{l_{2}^{2} E_{1}^{*}+2 l_{1}^{2} E_{2}^{*}}
\end{aligned}
$$

\section{ANALYTICAL PREDICTIONS AND COMPARISON WITH EXPERIMENTS}

So far, experimental results regarding the variation of the gap between solar cells have been interpreted in the literature by considering the deformations of the glass superstrate and the silicon cells only [2] There, the contributions of the backsheet and of the EVA were neglected, so that the following approximate formula was proposed [2] 


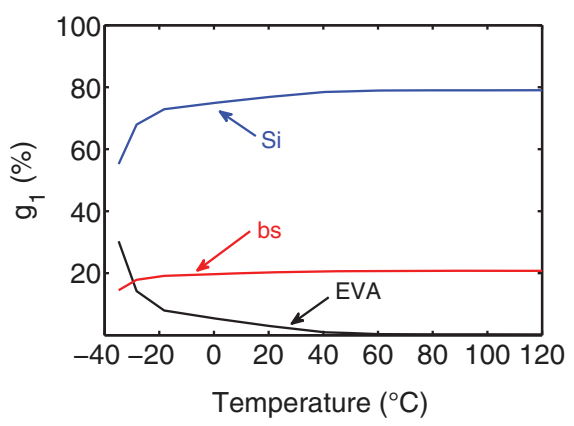

(a) Composite beam 1

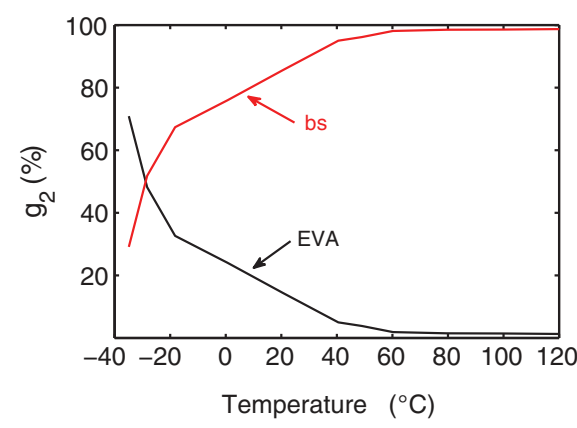

(b) Composite beam 2

Fig. 5 Weight factors for the thermal expansion coefficient; the labels (bs) and (Si) denote, respectively, the backsheet and the silicon cell

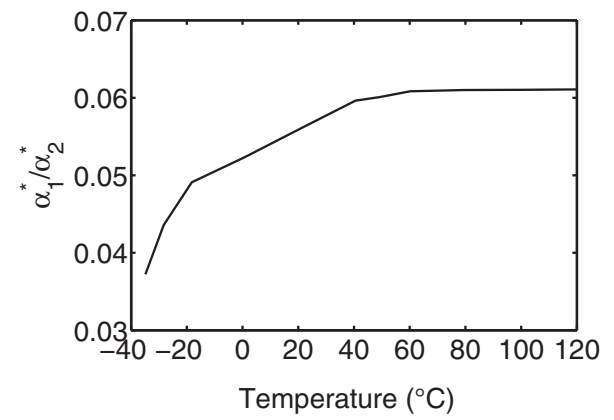

Fig. 6 Ratio between the composite thermal expansion coefficients of beam 1 and 2

$$
\Delta g=l_{\mathrm{G}}-l_{\mathrm{Si}}=\left(l_{G, 0}-2 l_{1,0}\right)+\left(l_{G, 0} \alpha_{\mathrm{G}}-2 l_{1,0} \alpha_{\mathrm{Si}}\right) \Delta T_{(15)}
$$

which suggests a linear dependence between $\Delta g$ and $\Delta T$. The predictions obtained using equation (15) are plotted in Fig. 7 with a black dashed line. Experimental measurements are also superimposed with black dots. The quadratic norm of the relative error between the numerical predictions and the experimental results is equal to 0.81 . A straightforward comparison between predictions and experiments shows that the simplified formula (15) slightly overestimates the gap variation for temperatures higher than $20^{\circ} \mathrm{C}$. On the other hand, a significant discrepancy between predictions and experiments is observed for temperatures below $0{ }^{\circ} \mathrm{C}$. The temperature-dependent behaviour of EVA becomes important for temperatures below zero and this may qualitatively explain the deviation from the linear trend predicted by equation (15). The contribution of EVA at low temperatures is in fact relevant in terms of stiffness for beam 2 (see Fig. 3), and in terms of thermal expansion coefficient for both beam 1 and beam 2 (see Fig. 5).

Let the general formula (14) now be considered, which allows different scenarios to be investigated towards the understanding of the role played by the different constituents on the deformability of PV modules, since all the materials are taken into account. The model predictions have been carried out for temperatures down to $-35^{\circ} \mathrm{C}$, consistently with the elastic modulus data of EVA plotted in Fig. 2. Lower temperatures were not explored, due to technical limitations in performing experimental measurements of the elastic modulus according to relaxation tests in a climate chamber at temperatures less than $-35^{\circ} \mathrm{C}$. In practical applications, however, the range of temperatures explored in the present study is exhaustive for the characterization of PV modules. In fact, the typical temperature range for $\mathrm{PV}$ modules in Europe is considered to lie between $-40^{\circ} \mathrm{C}$ and $85^{\circ} \mathrm{C}$.

Plotting the predictions of equation (14) versus $T$ in Fig. 7 (square dots), there is however a much stiffer response as compared to experiments, with a significantly lower predicted gap variation. The norm of the relative error between the analytical predictions and the experimental results is equal to 2.06. Here, it has to be remarked that equation (14) corresponds to the physical situation consisting in a perfect bonding between the interfaces of the stacks in beams 1 and 2, and between the two beams themselves. It also assumes that the cross-sections

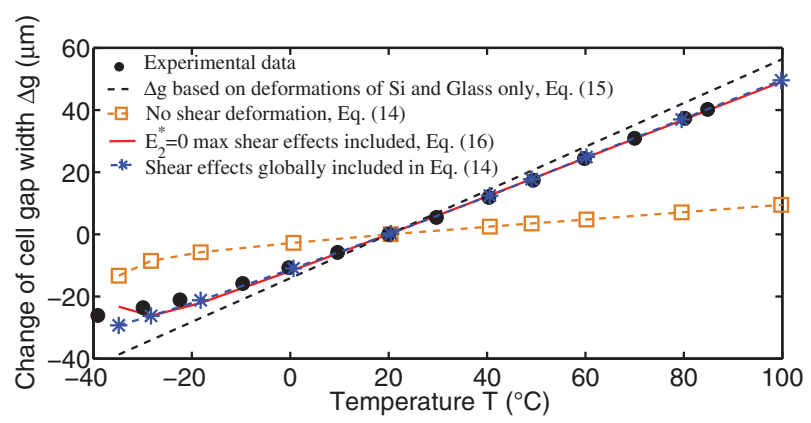

Fig. 7 Comparison between experimental results [2] and models' predictions 


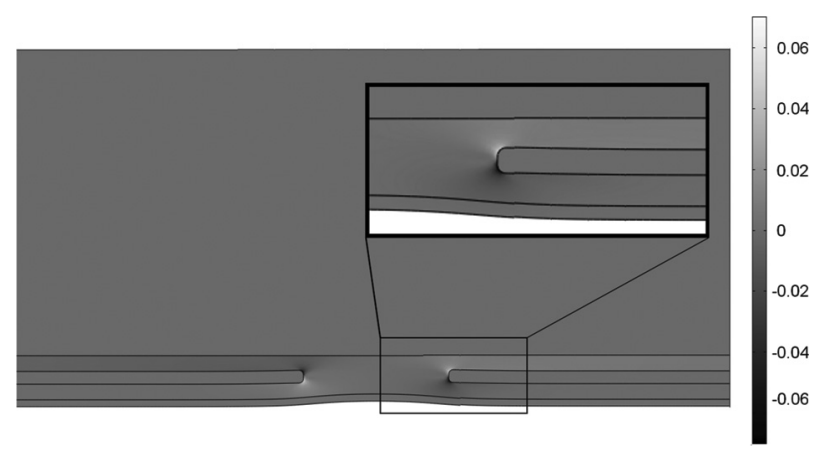

Fig. 8 Contour plot of shearing strains obtained from a 2D FE analysis, superimposed to the deformed geometry (amplification factor equal to 5); note the concentration of strains at the end of the Si cells and the non-planarity of beam 2 in the zoom of the central part

of the beams remain plane after deformation, neglecting shear deformations. This assumption allows the problem to be treated as a monodimensional one. To better interpret the experimental results, some of these constraints have to be released.

In experiments, the EVA layer deforms much more than silicon and the cross-section does not remain plane under deformation. To visualize the concentration of strains at the end of the Si cells, a 2D linear elastic finite element thermo-elastic analysis is performed with Comsol Multiphysics under plane strain. Periodic boundary conditions are considered on the vertical sides. The horizontal boundaries are stress-free. The reference temperature is set equal to $150^{\circ} \mathrm{C}$, which is the temperature at which bonding between the materials is made. The strain state at $T=-40^{\circ} \mathrm{C}$ is examined. The material parameters are those listed in Table 1. Regarding EVA, an elastic modulus of $1 \mathrm{GPa}$ is adopted. A nonlinear FE analysis with temperature-dependent properties was beyond the scope of the present study and is left for further investigation. The geometry is meshed with a triangular grid, with quadratic Lagrangian elements. A fine mesh around the interfaces was used to capture the strain concentration. Figure 8 shows the contour plot of the strain component $\gamma_{x y}$ at $T=-40^{\circ} \mathrm{C}$. It is superimposed to the deformed geometry of the PV module (with an amplification factor of 5) to show the non-planarity of the cross-sections, especially in beam 2. A zoom of that area is also provided for a better visualization.

The rigidity of the intermediate beam is mostly governed by backsheet (see Fig. 3). The de facto shearing of EVA drastically reduces the effective contribution of the backsheet to the stiffness of beam 2, so the stiffness of beam 2 becomes negligible as compared to that of beam 1 . These experimental observations suggest modifying equation (14) by setting $E_{2}^{*}=0$ to investigate this limit case

$$
\Delta g^{E_{2}^{*} \rightarrow 0}=l_{\mathrm{G}}-l_{2,0}-2 l_{1}=\left(l_{G, 0} \alpha_{\mathrm{G}}-2 \alpha_{1}^{*} l_{1,0}\right) \Delta T
$$

Here, it is interesting to note that the prediction no longer depends on the composite thermal expansion coefficient of beam 2. The variation of gap between solar cells is therefore given by the difference between the deformation of the glass superstrate and the deformation of the beam 1. The predictions are shown with a solid line in Fig. 7. The agreement with experiments significantly improves with respect to the case where $E_{2}^{*} \neq 0$ (norm of the relative error equal to 0.23 ). For $\Delta T<0$, the deviation from linearity observed in experiments is correctly captured, since the temperature-dependent elastic modulus of EVA in beam 1 is considered in the analysis.

If the square dots and solid curves can be considered as the lower and upper bounds to the gap variation, the intermediate situations physically correspond to a finite shearing of EVA. Within the framework of the monodimensional model discussed here, this phenomenon globally manifests as a reduced contribution of the backsheet to the stiffness of the composite beams, similarly to the independent expansion of the glass. The stiffness of beam 2 is in fact governed by the backsheet at high temperatures and therefore $E_{\mathrm{bs}}$ is the only parameter that can be tuned to effectively improve the predictions. It cannot be excluded that the change of $E_{\mathrm{bs}}$ with respect to the declared value provided by the producer might be partially due to a possible temperature dependency of its properties. However, this effect seems to be unlikely.

To investigate the influence of the backsheet contribution, its elastic modulus is artificially reduced, reducing in this way its stiffness and thermal expansion weight factors, $f_{\mathrm{bs}}$ and $g_{\mathrm{bs}}$, respectively. It is necessary to search for the value of $E_{\mathrm{bs}}$ that minimizes the norm of the relative error between the model predictions and the experimental values of the gaps. Only the data for $T>20^{\circ} \mathrm{C}$ have been considered in this procedure. In that range, the gap is a linear function of the temperature and the above criterion just requires to change $E_{\mathrm{bs}}$ in order to match the slope of the $\Delta g$ versus $T$ experimental data. The obtained curve is shown in Fig. 7 with a dashed line and it has been obtained by setting $E_{\mathrm{bs}}^{\text {new }}=5 E_{\mathrm{bs}} / 1000$. The norm of the relative error for this case is the lowest and is equal to 0.14. Comparing these predictions with those 


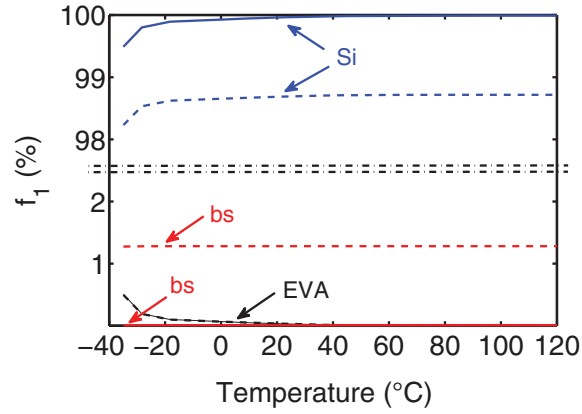

(a) Composite beam 1

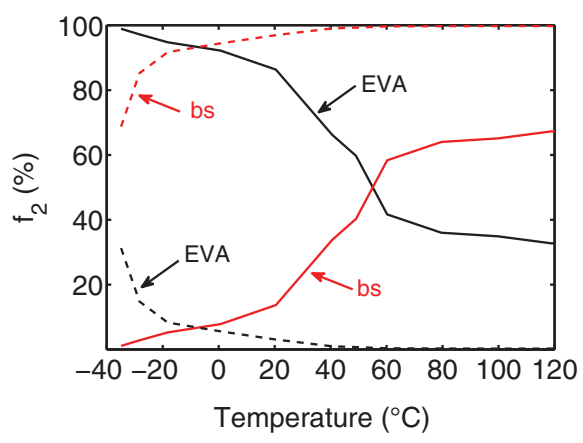

(b) Composite beam 2

Fig. 9 Weight factors for the composite Young's modulus after effectively considering the effect of shearing; dashed lines correspond to the predictions with neglected shear effects; the labels (bs) and (Si) denote, respectively, the backsheet and the silicon cell

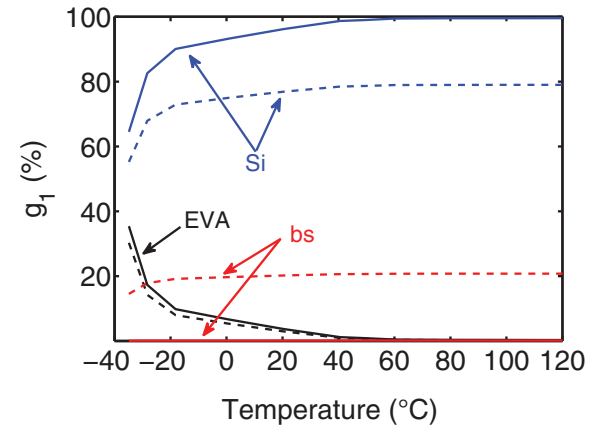

(a) Composite beam 1

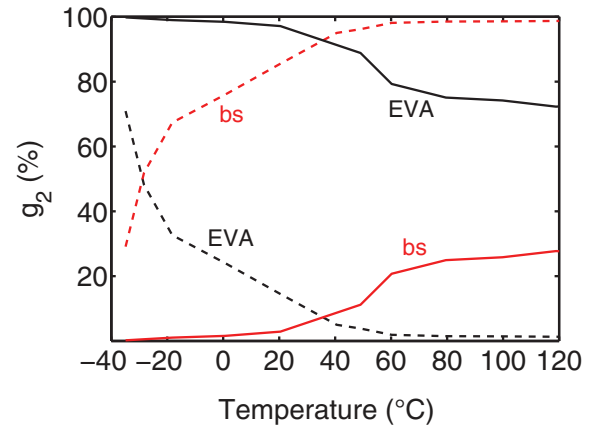

(b) Composite beam 2

Fig. 10 Weight factors for the thermal expansion coefficients after effectively considering the effect of shearing; dashed lines correspond to the predictions with neglected shear effects; the labels (bs) and (Si) denote, respectively, the backsheet and the silicon cell

obtained by applying equation (16) (solid line), note that they are both very close to experiments, apart from $T<-20^{\circ} \mathrm{C}$ where the solid line deviates from the experimental trend. The reason behind the search for an optimal solution using equation (14) is not to find the best agreement as possible with experiments, but rather to understand the reasons for the discrepancy between the predictions provided by equation (14) (considering all the materials, square dots line in the figure) and the experimental trend. From the present analysis it is possible to conclude that the stiffness of the backsheet plays an important role in the monodimensional model.

The corresponding updated weight factors are shown in Figs 9 and 10 with a solid line. The weight factors of the original model without taking account shearing effects are also superimposed with a dashed line. Regarding the composite elastic moduli, note that the stiffness of beam 1 is largely governed by the silicon cell, which has a weight factor approximately equal to 100 percent, see Fig. 9(a). On the other hand, the situation significantly changes for beam 2, where now the contribution of EVA prevails with respect to that of the backsheet for temperatures lower than $60^{\circ} \mathrm{C}$, see Fig. 9(b).

Looking at the composite thermal expansion coefficient of beam 1 (Fig. 10(a)), note that the contribution of backsheet is now sensibly reduced, whereas that of EVA remains almost unchanged. The silicon contribution is the prevailing one, especially for temperatures higher than $60^{\circ} \mathrm{C}$. As far as beam 2 is concerned, Fig. 10(b), the contribution of EVA is now prevailing over that of the backsheet in the whole temperature range. For temperatures lower than $20^{\circ} \mathrm{C}$, the weight factor of EVA reaches 100 per cent.

\section{DISCUSSION AND CONCLUSION}

In the present paper, a model based on multilayered beam theory has been proposed for the thermoelastic analysis of PV modules. Special emphasis has been given to the computation of the variation of the gap between solar cells when exposed to 
environmental temperatures, as happens during service conditions. This quantity is of paramount importance to assess the integrity of the electric connection between solar cells.

As compared to classical multilayered beams, the complexity of the problem is herein represented by the non-continuity of solar cells along the longitudinal direction, and by the temperature-dependent elastic properties of EVA. The comparison with experiments shows that it is reasonable to consider the stacks composed by EVA, solar cells, and backsheet as disconnected from the cover glass, whose effect can be regarded as a boundary condition on the deformation of the system. Two limit situations have then been modelled, one corresponding to a pure 1D model without shear deformation, and another with maximum effect of shear deformation obtained by setting $E_{2}^{*}=0$. They provide, respectively, the lower and the upper bounds to the variation of the gap between solar cells. Intermediate configurations with finite shearing of EVA can be effectively modelled by reducing the elastic modulus of backsheet, in order to reduce its contribution to the overall composite material properties of beams 1 and 2. The experimental data are best reproduced if the contribution of backsheet is significantly reduced. This suggests that, due to the shearing of EVA, the influence of the backsheet on the variation of the cell gap is negligible with a reasonable degree of accuracy. On the other hand, the temperature-dependent elastic modulus of EVA has an important role for the prediction of the stiffness of the composite beams and for capturing the deviation from linearity observed for temperatures below zero.

The analysis carried out for improving the predictions of equation (14) is important for two main reasons. First, in principle, the use of the complete solution including all the materials is expected to be easier to be generalized for the case of other geometries and materials. In fact, although setting $E_{2}^{*}=0$ was a reasonable approximation in the present application, this assumption should be checked with care if other materials are used. Second, improving the predictions of equation (14) has permitted the authors to understand that the elastic modulus of backsheet is a key parameter for the use of the present monodimensional beam model.

Although the degree of accuracy of the proposed model is satisfactory from the engineering point of view, more refined investigations based on $2 \mathrm{D}$ finite element models could be pursued. The main advantage of a 2D model concerns the possibility to simulate the non-planarity of the beam cross-section after deformation, an aspect that cannot be captured using a monodimensional model. It also makes it possible to obtain a detailed description of the local stress and strain fields. However, the present analytical approach provides useful information about the global behaviour of the PV module through weight factors that cannot be easily deduced from a finite element simulation. These weight factors can be used to assess the effect of different materials and layers arrangements on the stiffness and thermal expansion distributions of newly designed PV modules.

\section{FUNDING}

MP would like to thank the Alexander von Humboldt Foundation for supporting his research fellowship during the year 2010 at the Institut für Kontinuumsmechanik, Leibniz Universität Hannover (Germany). SKS and UE acknowledge financial support by the state of Lower Saxony, Germany.

(C) Authors 2011

\section{REFERENCES}

1 EPIA. Global market outlook for photovoltaics until 2014. European Photovoltaic Industry Association Technical Report, May 2010.

2 Eitner, U., Köntges, M., and Brendel, R. Use of digital image correlation technique to determine thermomechanical deformations in photovoltaic laminates: measurements and accuracy. Solar Energy Mater. Solar Cells, 2010, 94, 1346-1351.

3 Osterwald, C. R. and McMahon, T. J. History of accelerated and qualification testing of terrestrial photovoltaic modules: A literature review. Prog. Photovoltaics, 2009, 17, 11-33.

4 IEC 61215:2005. Crystalline silicon terrestrial photovoltaic (PV) modules - Design qualification and type approval. International Electrochemical Commission, 2005.

5 Timoshenko, S. P. Analysis of bi-metal thermostats. J. Opt. Soc. Am., 1925, 11, 233-255.

6 Suhir, E. Stresses in bi-metal thermostats. ASME J. Appl. Mech., 1986, 53, 657-660.

7 Suhir, E. Interfacial stresses in bimetal thermostats. ASME J. Appl. Mech., 1989, 56, 595-600.

8 Jiang, Q., Huang, Y., and Chandra, A. Thermal stresses in layered electronic assemblies. ASME J. Electron. Packaging, 1997, 119, 127-132.

9 Tsai, M. Y., Hsu, C. H., and Han, C. N. A note on Suhir's solution of thermal stresses for a die-substrate assembly. ASME J. Electron. Packaging, 2004, 126, 115-119.

10 Wang, K., Huang, Y., Chandra, A., and Hu, K. X. Interfacial shear stresses, peeling stresses, and die cracking stress in trilayer electronic assemblies. In Proceedings of the 7 th Intersociety Conference 
on Thermal and thermomechanical phenomena in electronic systems (ITHERM), 2000, vol. 2, pp. 56-64.

11 Wen, Y. and Basaran, C. Thermal stress analysis of multilayered microelectronic packaging. ASME J. Electron. Packaging, 2003, 125, 115-119.

12 Wen, Y. and Basaran, C. An analytical model for thermal stress analysis of multi-layered microelectronic packaging. Mech. Mater., 2004, 36, 369-385.

13 Carpinteri, A. and Paggi, M. Thermo-elastic mismatch in nonhomogenous beams. J. Eng. Math., 2008, 61, 371-384.

14 Brueckner, R. Mechanical properties of glasses. In Materials science and technology: a comprehensive treatment, vol. 9, glasses and amorphous materials (Eds R. W. Cahn, P. Hansen, and E. T. Kramer), 1991 (VCH, New York).

15 Greenwood, J. C. Silicon in mechanical sensors. J. Phys. E: Sci. Instrum., 1988, 21, 1114-1128.

16 Eitner, U., Kajari-Schröder, S., Köntges, M., and Brendel, R. Non-linear mechanical properties of ethylene-vinyl acetate (EVA) and its relevance to thermomechanics of photovoltaic modules. In Proceedings of the 25th European Conference on Photovoltaic solar energy, 2010, pp. 4366-4368.

17 Lyon, K. G., Salinger, G. L., Swenson, C. A., and White, G. K. Linear thermal expansion measurements on silicon from 6 to 340 K. J. Appl. Phys., 1977, 48, 865-868.

18 Voigt, W. Über die Beziehung zwischen den beiden Elastizitätskonstanten isotroper Körper. Wied. Ann., 1889, 38, 573-587.

\section{APPENDIX}

\section{Notation}

$E_{\mathrm{bs}} \quad$ elastic modulus of backsheet

$E_{\mathrm{bs}}^{\text {new }} \quad$ actual elastic modulus of backsheet

$E_{\mathrm{EVA}} \quad$ elastic modulus of the encapsulating polymer layer

$E_{\mathrm{G}} \quad$ elastic modulus of glass superstrate

$E_{\mathrm{j}} \quad$ elastic modulus of the $j$ th layer

$E_{\mathrm{Si}} \quad$ elastic modulus of silicon cell

$E_{\mathrm{i}}^{*} \quad$ composite elastic modulus of the $i$ th beam

$f_{i}^{\mathrm{bs}} \quad$ stiffness weight factor of backsheet for the $i$ th beam

\begin{tabular}{|c|c|}
\hline$f_{i}^{\text {EVA }}$ & $\begin{array}{l}\text { stiffness weight factor of EVA for the } i \text { th } \\
\text { beam }\end{array}$ \\
\hline$f_{1}^{S i}$ & $\begin{array}{l}\text { stiffness weight factor of silicon cell for } \\
\text { the beam } 1\end{array}$ \\
\hline$g_{i}^{\mathrm{bs}}$ & $\begin{array}{l}\text { thermal expansion weight factor of back- } \\
\text { sheet for the } i \text { th beam }\end{array}$ \\
\hline$g_{i}^{\mathrm{EVA}}$ & $\begin{array}{l}\text { thermal expansion weight factor of EVA } \\
\text { for the } i \text { th beam }\end{array}$ \\
\hline$g_{1}^{S i}$ & $\begin{array}{l}\text { thermal expansion weight factor of silicon } \\
\text { cell for the beam } 1\end{array}$ \\
\hline$l_{\mathrm{G}}$ & $\begin{array}{l}\text { span of the glass superstrate for a given } \\
\Delta T\end{array}$ \\
\hline$l_{\mathrm{G}, 0}$ & $\begin{array}{l}\text { reference span of the glass superstrate for } \\
\Delta T=0\end{array}$ \\
\hline$l_{i}$ & span of the $i$ th beam for a given $\Delta T$ \\
\hline$l_{i, 0}$ & reference span of the $i$ th beam for $\Delta T=0$ \\
\hline$N_{i}$ & axial force in the $i$ th beam \\
\hline$h_{\mathrm{bs}}$ & thickness of backsheet \\
\hline$h_{\mathrm{EVA}}$ & $\begin{array}{l}\text { thickness of the encapsulating polymer } \\
\text { layer }\end{array}$ \\
\hline$h_{\mathrm{G}}$ & thickness of the glass superstrate \\
\hline$h_{\mathrm{j}}$ & thickness of the $j$ th layer \\
\hline$h_{\mathrm{Si}}$ & thickness of silicon solar cell \\
\hline$h$ & total thickness of PV laminate \\
\hline$t$ & beam thickness \\
\hline$x_{i}$ & $\begin{array}{l}\text { equilibrium coordinates of the } i \text { th } \\
\text { interface }\end{array}$ \\
\hline
\end{tabular}

coefficient of thermal expansion of the backsheet

coefficient of thermal expansion of the encapsulating polymer layer

coefficient of thermal expansion of the glass superstrate

coefficient of thermal expansion of the $j$ th layer

composite coefficient of thermal expansion of the $i$ th beam

variation of the gap between solar cells temperature variation from $20^{\circ} \mathrm{C}$ total potential energy of the system axial strain axial strain in the $i$ th beam axial stress in the $j$ th layer 